\title{
Gene microarray analysis of the circular RNAs expression profile in human gastric cancer
}

\author{
YONGHUA SHEN $^{1 *}$, JUANJUAN ZHANG $^{2 *}$, ZIYI FU $^{3}$, BIN ZHANG $^{1}$, \\ MIN CHEN ${ }^{1}$, XIUFENG LING $^{2}$ and XIAOPING ZOU ${ }^{1}$ \\ ${ }^{1}$ Department of Gastroenterology, The Affiliated Drum Tower Hospital of Nanjing University, \\ Medical School, Nanjing, Jiangsu 210008; ${ }^{2}$ Department of Reproduction; \\ ${ }^{3}$ Nanjing Maternal and Child Health Medical Institute, Affiliated Nanjing Maternal and Child Health Hospital, \\ Nanjing Medical University, Nanjing, Jiangsu 210004, P.R. China
}

Received August 13, 2017; Accepted April 3, 2018

DOI: $10.3892 / \mathrm{ol} .2018 .8590$

\begin{abstract}
Human gastric cancer is a common malignant neoplasm of the digestive system and represents a threat to human health worldwide. The mechanisms underlying gastric cancer germination and development are not yet fully understood. Circular RNAs (circRNAs) serve crucial roles in various physiological and pathological processes, particularly cancer. However, few studies have focused on the mechanisms involving circRNAs in gastric cancer. Therefore the present study set out to identify the differentially expressed circRNAs in gastric cancer. Three specimens of gastric cancer and normal gastric tissue were selected and circRNA microarray analysis was performed to detect the differentially expressed circRNAs. The changes in circRNAs were confirmed by reverse transcription-quantitative polymerase chain reaction analysis. A total of 347 upregulated and 603 downregulated circRNAs (fold-change, $>2.0$ ) were identified in gastric cancer compared with the normal gastric tissue. A total of 20 selected circRNAs were dysregulated during gastric cancer, which suggests their potential role in gastric cancer. The present study identified circRNAs in the expression profile of human gastric cancer that were potentially involved in the underlying molecular mechanisms of its development.
\end{abstract}

Correspondence to: Professor Xiufeng Ling, Department of Reproduction, Affiliated Nanjing Maternal and Child Health Hospital, Nanjing Medical University, 123 Mochou Road, Nanjing, Jiangsu 210004, P.R. China

E-mail: lingxiufeng_njfy@163.com

Professor Xiaoping Zou, Department of Gastroenterology, The Affiliated Drum Tower Hospital of Nanjing University, Medical School, 321 Zhongshan Road, Nanjing, Jiangsu 210008, P.R. China E-mail: 13770771661@163.com

*Contributed equally

Key words: gastric cancer, circular RNA, microarray analysis

\section{Introduction}

Human gastric cancer is the fourth most common malignancy and the second leading cause of cancer-related deaths worldwide (1). Adenocarcinoma is the most common pathological type of gastric cancer, while lymphoma, carcinoid, and sarcoma constitute $<5 \%$ of the pathology (2). The pathogenesis of gastric cancer is complicated owing to the interaction of multiple factors, including Helicobacter pylori infection, environment, and heredity. The environmental factors play critical roles in the pathogenesis of gastric cancer; the major risk factors include smoking and diet (3). However, determining the molecular markers of gastric cancer is yet a great challenge.

Recently, the identification and characterization of circular RNA (circRNA) have revolutionized the field of RNA. CircRNAs have gained increasing attention in deciphering the complicated mechanisms underlying the malignant processes such as tumorigenesis, multidrug resistance, invasion, and metastasis. Although circRNAs have been reported as early as 20 years ago (4), they were mostly misinterpreted as splicing artefacts or gene rearrangements. Following high-throughput RNA sequencing and bioinformatics, thousands of different circRNAs have been rediscovered in the recent several years (5-8). Preliminary data revealed that circRNAs were abundantly expressed and evolutionarily conserved across the eukaryotes and functioned as miRNA sponges $(5,6,9)$. Cdr1as (also known as ciRS-7), as the maximally studied circRNA, was reported as the miR-7 sponge or inhibitor $(5,6)$.

ciRS-7 was highly expressed in a wide variety of cancer cell lines, and ciRS-7/miR-7 network suggested a therapeutic potential for carcinoma. This network might regulate the majority of the cancer pathways such as p21-activated kinase 1 (Pak1) (10), epidermal growth factor receptor (EGFR) (11), activated cdc42-associated kinase 1 (Ack1) (12), and phosphoinositide 3-kinase catalytic subunit delta (PIK3CD) (13). Increasing number of evidence indicated miR-7 as a potential tumor suppressor in several human cancers. Xiong et al reported that miR-7 selectively induced growth suppression and apoptosis of non-small cell lung cancer (NSCLC) by targeting B-cell lymphoma-2 (BCL-2) in vitro (14). Similarly, miR-7 was confirmed as a novel miRNA exhibiting tumor 
suppression function in colon cancer (15). Although the majority of reports supported the tumor-enhancing effect of circRNAs, the converse was also reported. ciR-ITCH demonstrated an inhibitory effect on esophageal squamous cell carcinoma, acting as a sponge of miR-7, miR-17, and miR-214 via the regulation of the Wnt/ $\beta$-catenin pathway (16). Altogether, these results suggested that the relationship between circRNA and cancer was complicated and precise mechanisms needed further investigation.

In this study, we presented the circRNAs' expression profile in normal gastric tissue and gastric adenocarcinoma through microarray technology in order to explore the function of circRNAs in gastric cancer for early diagnosis and treatment of cancer.

\section{Materials and methods}

Patient samples. The present study was approved by the Research Ethics Committee of the Affiliated Drum Tower Hospital of Nanjing University, Medical School, and all patients provided informed consent before the samples were collected. Gastric cancer was confirmed by histopathological diagnosis. Finally, 15 patients (eight men, seven women; mean age 64.1 years, range 48-81) were enrolled and 15 pairs of gastric carcinoma tissues and normal para-carcinoma samples were collected. All samples were rapidly frozen in liquid nitrogen and stored at $-80^{\circ} \mathrm{C}$ for subsequent investigation. For circRNA microarray analysis, a total of 3 gastric carcinoma and 3 normal para-carcinoma gastric tissues (control) were randomly selected for the study.

RNA extraction. Total RNA was extracted from the frozen tissue block that was homogenized (IKA Werke GmbH \& Co. KG, Staufen, Staufen, Germany) and resuspended in TRIzol reagent (Invitrogen, Carlsbad, CA, USA), according to the manufacturer's instructions. Then, the total RNA was quantified using a NanoDrop ND-1000 spectrophotometer (NanoDrop Technologies, Wilmington, DE, USA). Additionally, the RNA integrity was assessed by denaturing agarose gel electrophoresis.

RNA labeling and array hybridization. The sample labeling and microarray hybridization were performed by KangChen Biotech (Shanghai, China). Briefly, the circRNA was treated with RNase R (Epicenter Biotechnologies, USA) to remove the linear RNAs. Each sample was amplified and transcribed into fluorescent cRNA using a random priming method (Arraystar Super RNA Labeling kit; Arraystar Inc., Rockville, MD, USA). Subsequently, these labeled cRNAs were purified by RNeasy Mini kit (cat. no. 74106, Qiagen $\mathrm{GmbH}$, Hilden, Germany Germany), and the concentration and specific activity were measured by NanoDrop (NanoDrop Technologies). Then, $1 \mu \mathrm{g}$ of each labeled cRNA was fragmented by adding $5 \mu \mathrm{l}$ of $10 \mathrm{X}$ blocking agent and $1 \mu \mathrm{l}$ of $25 \mathrm{X}$ fragmentation buffer, followed by heating at $60^{\circ} \mathrm{C}$ for $30 \mathrm{~min}$. Then, $25 \mu \mathrm{l}$ of $2 \mathrm{X}$ hybridization buffer was added to dilute the labeled cRNA, and $50 \mu \mathrm{l}$ of the hybridization solution was dispensed into the gasket slide and assembled on the circRNA expression microarray slide. These slides were incubated for $17 \mathrm{~h}$ at $65^{\circ} \mathrm{C}$ in a hybridization oven (Agilent).

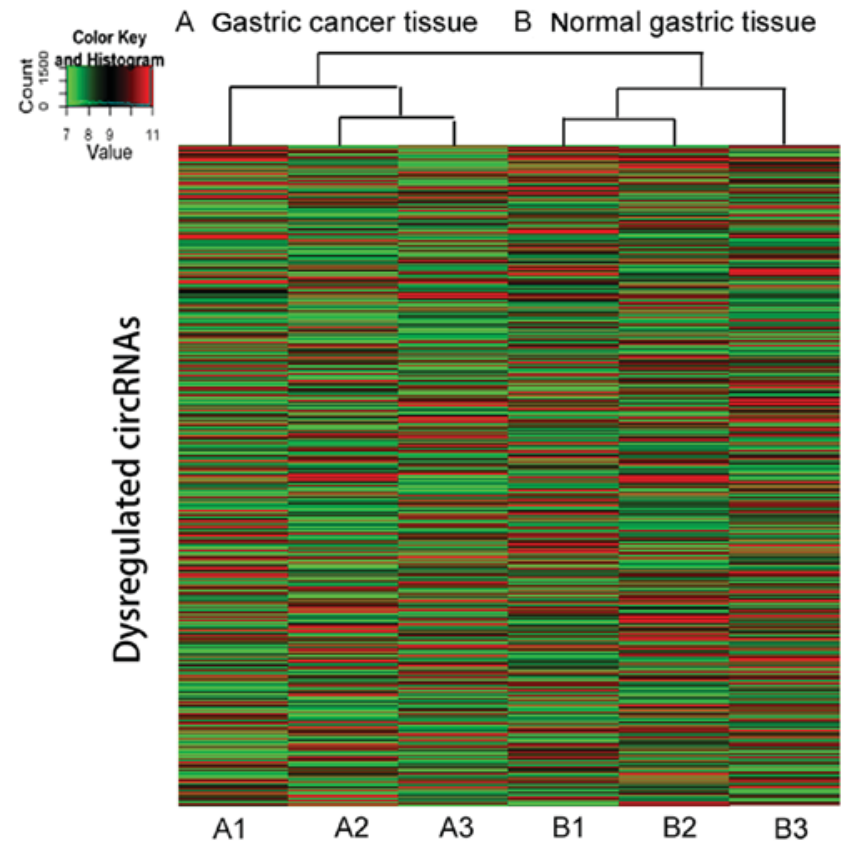

Figure 1. Differentially expressed circRNAs between (A) gastric cancer tissue and (B) adjacent normal gastric tissue. The result from hierarchical clustering analysis reveals circRNA expression variation among samples. The color scale reflects the $\log _{2}$ signal intensity and runs from green (low intensity), to black (medium intensity), to red (strong intensity). circRNA, circular RNA.

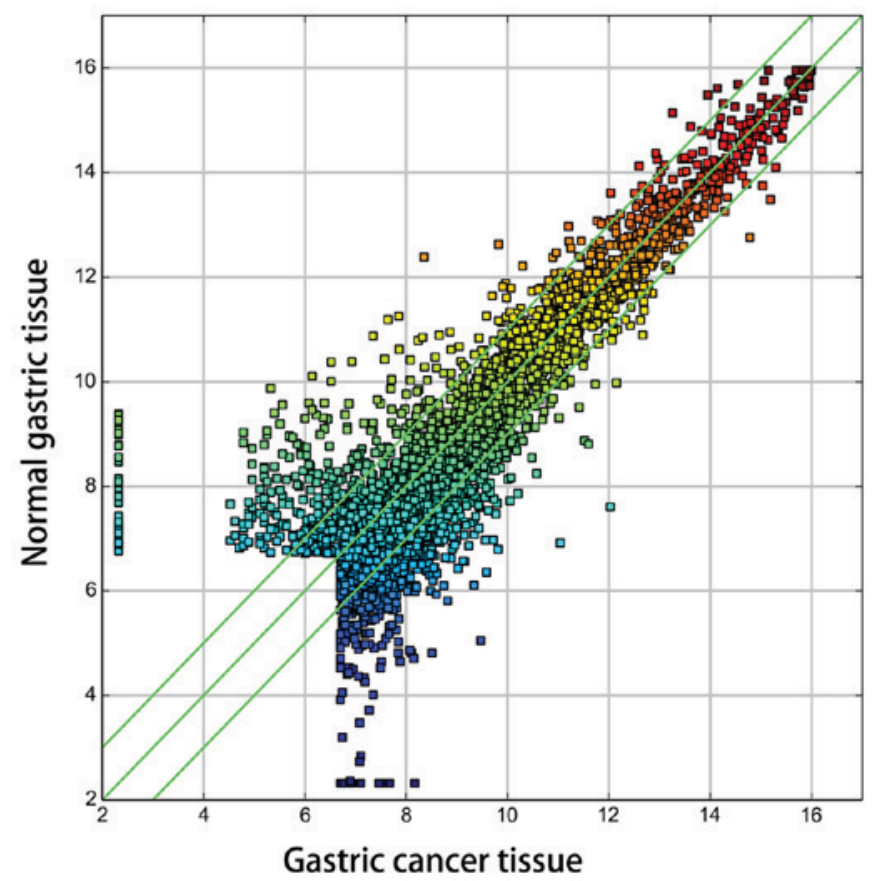

Figure 2. Scatter-plot of circRNA expression variation between the gastric cancer tissue (x-axis) and normal gastric tissue (y-axis). The values of the $\mathrm{x}$-axis and $\mathrm{y}$-axis in the Scatter-plot are the averaged normalized signal values of gastric cancer and normal gastric tissues ( $\log _{2}$ scaled). The green lines are fold-change lines. CircRNAs in the Scatter-plot above the top green line and below the bottom green line indicate more than 2.0 fold-change of circRNAs between the two compared samples. circRNA, circular RNA.

The hybridized arrays were washed, fixed, and scanned using the Axon GenePix 4000B microarray scanner (Molecular Devices, LLC, Sunnyvale, CA, USA). 

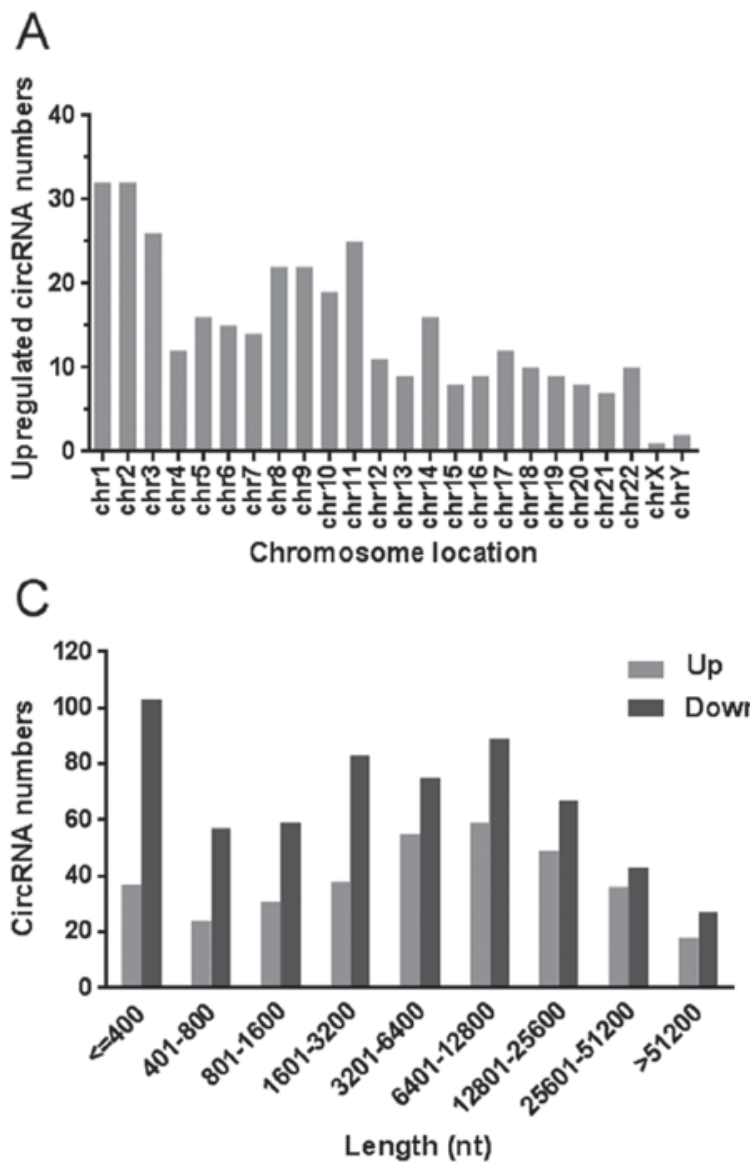

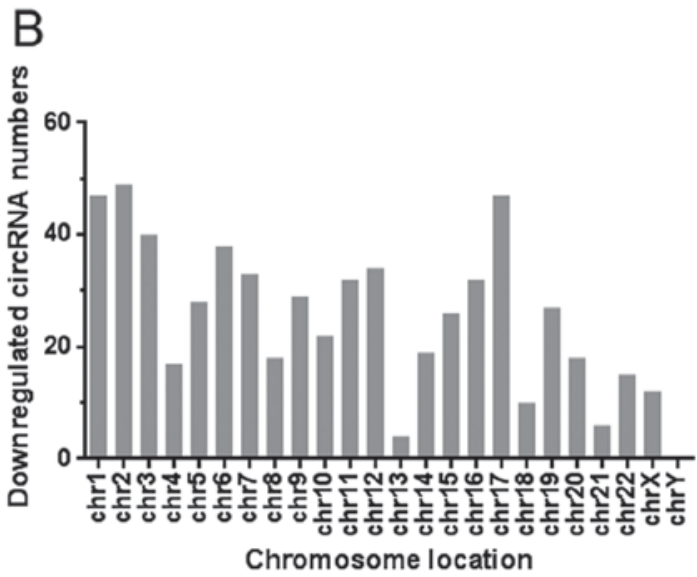

$\mathrm{D}$

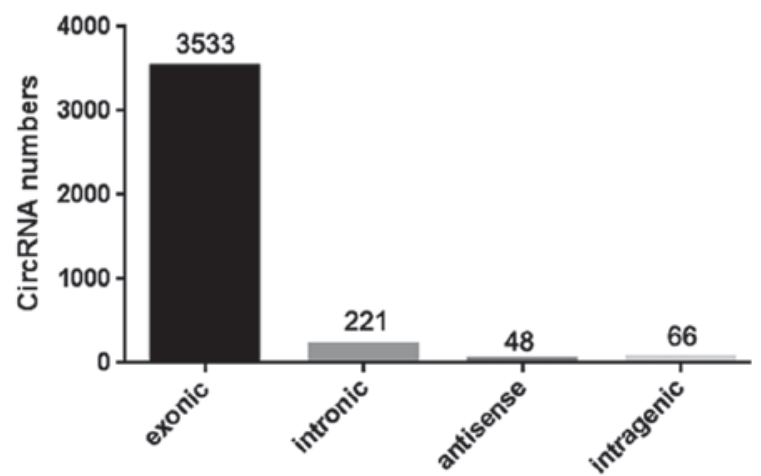

Figure 3. Annotation of differentially expressed circRNAs in gastric cancer tissues. (A) The location of upregulated circRNAs in human chromosomes. (B) The location of downregulated circRNAs in human chromosomes. (C) The length distribution of the dysregulated circRNAs. (D) The functional classification between the dysregulated circRNAs and their targets. circRNA, circular RNA.

Microarray analysis. The Arraystar Human Circular RNA Microarray V2.0 (Arraystar, Inc.) was designed for the purpose of profiling both circRNAs and protein-coding RNAs in the human genome. The differentially expressed circRNAs were identified through fold-change filtering and standard Student's t-test. The circRNAs are exhibiting a fold-change $\geq 2.0$ and a P-value $<0.05$ were selected as significantly differentially expressed circRNAs.

Quantitative reverse transcription PCR. cDNA samples were prepared from total RNA of gastric tissues by reverse transcription. In total, 20 circRNAs were analyzed by SYBR Green I dye-based detection with specific primer sequences. The primer sequences were shown in Table I. The $2^{-\Delta \Delta \mathrm{Cq}}$ method was performed in 18 samples and applied for the quantification of the relative expression of circRNAs that was normalized against the expression of the housekeeping gene, GAPDH.

Statistical analysis. The two groups were compared by the standard Student's t-test for the evaluation of the microarray analysis. The results were considered statistically significant at a P-value $<0.05$. The false discovery rate (FDR) was calculated to correct the P-value. Fold-change $\geq 2.0$ and P-value $<0.05$ were used to identify the differentially expressed circRNAs.

\section{Results}

circRNA expression profiles in gastric cancer tissues relative to adjacent normal gastric tissues. Randomly, 3 gastric cancer and 3 adjacent normal gastric tissues were selected for a standard circRNA microarray independently. The circRNA expression patterns between gastric cancer and the adjacent normal gastric tissues were found to be significantly different. After scanning and normalization, a total of 950 circRNAs were found to be differentially expressed in the microarray (fold-change in expression $\geq 2.0, \mathrm{P}<0.05$ ), consisting of 347 upregulated circRNAs and 603 downregulated circRNAs (data not shown). The hierarchical clustering of circRNA expression described the variation in the expression between the groups of gastric cancer and normal gastric tissues (Fig. 1). Furthermore, the variation in circRNA expression among the samples was assessed by Scatter-plot visualization (Fig. 2).

Annotation of differentially expressed circRNAs in gastric cancer tissues. The general data, including the chromosome location, length distribution and functional classification of these differentially expressed circRNAs were summarized. Fig. 3A and B demonstrated that the up- and downregulated circRNAs were located in human chromosomes. The length data displayed two peaks that were distributed among these 
Table I. Primer sequences for reverse transcription-polymerase chain reaction.

\begin{tabular}{|c|c|c|}
\hline Gene & Direction & Primer sequence \\
\hline \multirow[t]{2}{*}{ hsa_circ_0001017 } & Forward & AGTGCGAAGTAATCTATGCCAGC \\
\hline & Reverse & AGCCATTCTTTGCTGGGCTC \\
\hline \multirow[t]{2}{*}{ hsa_circ_0001772 } & Forward & GCCAGAGGAGGAGCAGCTTTA \\
\hline & Reverse & GCTCTTCATCTGACAAATCCGAC \\
\hline \multirow[t]{2}{*}{ hsa_circ_0002346 } & Forward & GTGCAAACCAGTTTTCGGCG \\
\hline & Reverse & TCCAGTTCTCATCTTGTTGGCA \\
\hline \multirow[t]{2}{*}{ hsa_circ_0000072 } & Forward & TTGGCAGCAAATGGAGTTCGT \\
\hline & Reverse & GTGCCTGCCACCATTTCCTTA \\
\hline \multirow[t]{2}{*}{ hsa_circ_0003221 } & Forward & GATGCGGGGCAATGCACTA \\
\hline & Reverse & ACCAGTACCCAGGTGAGTCTT \\
\hline \multirow[t]{2}{*}{ hsa_circ_0001865 } & Forward & GCTCCACAGACTTCCCAGAGT \\
\hline & Reverse & GGCAAGTTCCAACGTCTCCT \\
\hline \multirow[t]{2}{*}{ hsa_circ_0003441 } & Forward & ACCACAGTTCTTGGTGGTGAAG \\
\hline & Reverse & TGACTTTGTCTGGAGAGCTTGTG \\
\hline \multirow[t]{2}{*}{ hsa_circ_0008285 } & Forward & GCTGTTAACGGGAAAGGTTGAA \\
\hline & Reverse & GCGTCTGTTGAAGTCGTGGA \\
\hline \multirow[t]{2}{*}{ hsa_circ_0023923 } & Forward & AGCACATCAAAGCTGCCCAA \\
\hline & Reverse & TGCACTGAATTAAGTCTCCCCA \\
\hline \multirow[t]{2}{*}{ hsa_circ_0000347 } & Forward & GAAAAAGAACCAATGCAAAGAAGGT \\
\hline & Reverse & GCACTGAATTAAGTCTCTGCAACT \\
\hline \multirow[t]{2}{*}{ hsa_circ_0046881 } & Forward & AAGTCAGGCAGCTTTGCTGG \\
\hline & Reverse & CACAGTTGGTTAGCCACAGC \\
\hline \multirow[t]{2}{*}{ hsa_circ_0023940 } & Forward & ATGCTCCTGTTCAAAGATGCCA \\
\hline & Reverse & TTTGAAGACCACCACCCAACT \\
\hline \multirow[t]{2}{*}{ hsa_circ_0023891 } & Forward & CCTGCTACTACACCAACAGGC \\
\hline & Reverse & ACTGAATTAAGTCTGTGCTCCTGA \\
\hline \multirow[t]{2}{*}{ hsa_circ_0002433 } & Forward & TGAGCGTTTTATTCAGTATTTGGCT \\
\hline & Reverse & GCACTGAATTAAGTCTTGCAATCCA \\
\hline \multirow[t]{2}{*}{ hsa_circ_0050278 } & Forward & AAGCCAGACCTGATCACTTGTC \\
\hline & Reverse & TGTCAATGGTCCCTGTGGGT \\
\hline \multirow[t]{2}{*}{ hsa_circ_0000154 } & Forward & ACCAACGTTGAGCAAGATGC \\
\hline & Reverse & TTCTCCAGTGTCATTCCAACAGA \\
\hline \multirow[t]{2}{*}{ hsa_circ_0075048 } & Forward & GGCCACATCGACAACTCCAT \\
\hline & Reverse & GCTCGTTCACACTTGTTGATGC \\
\hline \multirow[t]{2}{*}{ hsa_circ_0001824 } & Forward & TGCATCAGCTCCAGGGCAAT \\
\hline & Reverse & TTGAAAGAAATGTGGCATGTGAGA \\
\hline \multirow[t]{2}{*}{ hsa_circ_0000835 } & Forward & CAGCATGGTCATGGAGGATGG \\
\hline & Reverse & ATGCTTGATGCCTATTGCCACT \\
\hline \multirow[t]{2}{*}{ hsa_circ_0000825 } & Forward & GAAAAGCGCGCTAAAGCTGA \\
\hline & Reverse & TCCATCTCAGCACGGAGTTCA \\
\hline \multirow[t]{2}{*}{ hsa_circ_0009109 } & Forward & ATCTGGCTCAGATGACACCAA \\
\hline & Reverse & TATGTTTGCTCGGTGCCCTG \\
\hline \multirow[t]{2}{*}{ hsa_circ_0087855 } & Forward & ACTTCCACACCTGCATCCAT \\
\hline & Reverse & TGCTTTCACCTGTCAGTTGCT \\
\hline hsa_circ_0001747 & Forward & GACAAGCTGGTGTTGAAGGGT \\
\hline & Reverse & AGCAGGCCTTTCGAGCTTTAG \\
\hline hsa_circ_0009061 & Forward & CCAAGCATCAGGTGTGGAGG \\
\hline & Reverse & TCTCTGTACTCTACTGTGCGGT \\
\hline hsa_circ_0000997 & Forward & TGCACCACTGGATGTTGTTTACT \\
\hline & Reverse & GTGGTCTCCACCTGTTTTGGAT \\
\hline
\end{tabular}


Table I continued.

\begin{tabular}{lll}
\hline Gene & Direction & Primer sequence \\
\hline hsa_circ_0001073 & Forward & ACTTGTTCCAACTCAAGTGCTATAC \\
hsa_circ_0000085 & Reverse & GTAGCAAAACAATGCCGCCG \\
& Forward & TTTGGCAGACTTTTACCTGGTG \\
hsa_circ_0088021 & Reverse & TGGATTGCTGCTTAAGCTTCCT \\
hsa_circ_0020353 & Forward & GCTGAACAGGTGCCTGAACT \\
& Reverse & CAATTCCAGGTCTGCTGCCG \\
& Forward & GCAGACTCCTGCAAGTTCCC \\
\hline
\end{tabular}

Table II. Upregulated circRNAs between gastric cancer and normal gastric tissues.

\begin{tabular}{|c|c|c|c|c|c|c|c|c|c|}
\hline circRNA & P-value & $\mathrm{FC}$ & $\begin{array}{c}\log _{2} \\
\mathrm{FC}\end{array}$ & Regulation & $\begin{array}{l}\text { circRNA } \\
\text { type }\end{array}$ & Chrom & Strand & Best transcript & Gene symbol \\
\hline hsa_circRNA_104804 & $<0.001$ & 117.219 & 6.873 & Up & Exonic & $\operatorname{chr} 9$ & - & uc004amv.3 & UBQLN1 \\
\hline hsa_circRNA_102678 & $<0.001$ & 125.119 & 6.967 & Up & Exonic & $\operatorname{chr} 2$ & + & uc002rpd.3 & CRIM1 \\
\hline hsa_circRNA_100261 & 0.011 & 41.319 & 5.368 & Up & Exonic & $\operatorname{chr} 1$ & - & uc001dex.4 & ANKRD13C \\
\hline hsa_circRNA_100927 & 0.002 & 70.919 & 6.148 & Up & Exonic & $\operatorname{chr} 11$ & - & uc001pbl.3 & PICALM \\
\hline hsa_circRNA_100924 & 0.001 & 88.219 & 6.463 & Up & Exonic & $\operatorname{chr} 11$ & - & uc001pbl.3 & PICALM \\
\hline hsa_circRNA_100922 & $<0.001$ & 101.119 & 6.659 & Up & Exonic & $\operatorname{chr} 11$ & - & uc001pbl.3 & PICALM \\
\hline hsa_circRNA_102713 & 0.010 & 44.119 & 5.463 & $\mathrm{Up}$ & Exonic & $\operatorname{chr} 2$ & - & uc002rus.3 & SRBD1 \\
\hline hsa_circRNA_102499 & 0.008 & 56.519 & 5.820 & Up & Exonic & chr19 & + & uc002npf.3 & ZNF85 \\
\hline hsa_circRNA_102315 & 0.007 & 52.219 & 5.706 & Up & Exonic & $\operatorname{chr} 18$ & + & uc002ktp.3 & MIB1 \\
\hline hsa_circRNA_100911 & 0.004 & 71.319 & 6.156 & Up & Exonic & $\operatorname{chr} 11$ & - & uc001pbl.3 & PICALM \\
\hline hsa_circRNA_104850 & 0.015 & 45.319 & 5.502 & Up & Exonic & chr9 & + & uc011lwa.2 & RAD23B \\
\hline hsa_circRNA_100925 & 0.002 & 75.269 & 6.233 & $\mathrm{Up}$ & Exonic & $\operatorname{chr} 11$ & - & uc001pbl.3 & PICALM \\
\hline hsa_circRNA_102293 & 0.010 & 49.919 & 5.641 & Up & Exonic & $\operatorname{chr} 18$ & + & uc002knq. 2 & CCDC165 \\
\hline hsa_circRNA_102737 & $<0.001$ & 133.619 & 7.061 & Up & Exonic & $\operatorname{chr} 2$ & - & uc002sbj.3 & XPO1 \\
\hline hsa_circRNA_104868 & 0.021 & 34.519 & 5.109 & Up & Exonic & $\operatorname{chr} 9$ & - & uc010muc.1 & KIAA0368 \\
\hline hsa_circRNA_100381 & 0.013 & 48.619 & 5.603 & Up & Exonic & $\operatorname{chr} 1$ & + & uc001gev.3 & DCAF6 \\
\hline hsa_circRNA_104707 & $<0.001$ & 120.019 & 6.907 & Up & Exonic & chr8 & - & uc003yvs.3 & PTK2 \\
\hline hsa_circRNA_104532 & $<0.001$ & 132.219 & 7.046 & $\mathrm{Up}$ & Exonic & $\operatorname{chr} 7$ & + & uc003wme.3 & RBM33 \\
\hline hsa_circRNA_104492 & 0.012 & 45.319 & 5.502 & Up & Exonic & $\operatorname{chr} 7$ & + & uc003vqs.3 & MKLN1 \\
\hline hsa_circRNA_104689 & 0.009 & 53.719 & 5.747 & Up & Exonic & chr8 & - & uc003ysz.2 & ASAP1 \\
\hline hsa_circRNA_100380 & 0.010 & 56.219 & 5.812 & Up & Exonic & $\operatorname{chr} 1$ & + & uc001gev.3 & DCAF6 \\
\hline hsa_circRNA_104016 & 0.011 & 54.219 & 5.760 & Up & Exonic & $\operatorname{chr} 5$ & + & uc003mby.4 & ERGIC1 \\
\hline hsa_circRNA_100715 & 0.021 & 34.419 & 5.105 & Up & Exonic & $\operatorname{chr} 10$ & - & uc001lif.4 & СТВP2 \\
\hline hsa_circRNA_101270 & $<0.001$ & 108.219 & 6.757 & $\mathrm{Up}$ & Exonic & $\operatorname{chr} 13$ & + & uc001vib.4 & TDRD3 \\
\hline hsa_circRNA_102830 & 0.014 & 41.519 & 5.375 & Up & Exonic & $\operatorname{chr} 2$ & + & uc002twg.3 & ACVR2A \\
\hline hsa_circRNA_100241 & $<0.001$ & 123.419 & 6.947 & Up & Exonic & $\operatorname{chr} 1$ & - & uc001cyx.1 & OMA1 \\
\hline hsa_circRNA_104052 & 0.001 & 103.319 & 6.690 & Up & Exonic & chr6 & + & uc003mwi.3 & CDYL \\
\hline hsa_circRNA_102298 & 0.003 & 87.319 & 6.448 & Up & Exonic & $\operatorname{chr} 18$ & - & uc002kod.1 & PPP4R1 \\
\hline hsa_circRNA_100097 & 0.011 & 44.619 & 5.479 & $\mathrm{Up}$ & Exonic & $\operatorname{chr} 1$ & + & uc001bgi.2 & KDM1A \\
\hline
\end{tabular}

FC, fold change; chrom, chromosome; circRNA, circular RNA.

dysregulated circRNAs in $\leq 400 \mathrm{bp}$ and $1,601-25,600 \mathrm{bp}$ (Fig. 3C). Fig. 3D revealed the relationship between the mentioned circRNAs and their molecular targets including exonic, intronic, antisense and intragenic. Nevertheless, the exonic targets occupied the vast majority of all types of functional classification. 
Table III. Downregulated circRNAs between gastric cancer and normal gastric tissues.

\begin{tabular}{|c|c|c|c|c|c|c|c|c|c|}
\hline circRNA & P-value & $\mathrm{FC}$ & $\begin{array}{c}\log _{2} \\
\text { FC }\end{array}$ & Regulation & $\begin{array}{l}\text { circRNA } \\
\text { type }\end{array}$ & Chrom & Strand & Best_transcript & Gene symbol \\
\hline hsa_circRNA_103538 & $<0.001$ & -40.819 & -5.351 & Down & Exonic & $\operatorname{chr} 3$ & + & uc003fpi.3 & MAP3K13 \\
\hline hsa_circRNA_102016 & 0.038 & -8.032 & -3.005 & Down & Exonic & $\operatorname{chr} 17$ & - & uc002heo.1 & SSH2 \\
\hline hsa_circRNA_102464 & 0.006 & -17.362 & -4.117 & Down & Exonic & $\operatorname{chr} 19$ & + & uc002myp.3 & PKN1 \\
\hline hsa_circRNA_100109 & 0.036 & -8.135 & -3.024 & Down & Exonic & chr1 & + & uc001bmt.1 & ARID1A \\
\hline hsa_circRNA_103477 & 0.002 & -20.819 & -4.379 & Down & Exonic & $\operatorname{chr} 3$ & + & uc003eqt.3 & EPHB1 \\
\hline hsa_circRNA_100801 & 0.007 & -21.424 & -4.421 & Down & Exonic & $\operatorname{chr} 11$ & + & uc001mxq.4 & HSD17B12 \\
\hline hsa_circRNA_104984 & 0.028 & -12.154 & -3.603 & Down & Exonic & $\operatorname{chr} \mathrm{X}$ & - & uc004czk.2 & MAP3K15 \\
\hline hsa_circRNA_104968 & 0.030 & -9.387 & -3.230 & Down & Exonic & chr9 & + & uc004coa.3 & EHMT1 \\
\hline hsa_circRNA_101585 & 0.034 & -11.713 & -3.550 & Down & Exonic & chr15 & - & uc010biv.1 & CELF6 \\
\hline hsa_circRNA_102540 & 0.035 & -10.064 & -3.331 & Down & Exonic & $\operatorname{chr} 19$ & + & uc002ohk.3 & SIPA1L3 \\
\hline hsa_circRNA_103568 & 0.038 & -9.790 & -3.291 & Down & Exonic & $\operatorname{chr} 3$ & - & uc003fxp.2 & DLG1 \\
\hline hsa_circRNA_100144 & $<0.001$ & -57.519 & -5.845 & Down & Exonic & $\operatorname{chr} 1$ & + & uc001bui.3 & TXLNA \\
\hline hsa_circRNA_105041 & 0.005 & -27.519 & -4.782 & Down & Exonic & $\operatorname{chrX}$ & - & uc004flx.1 & G6PD \\
\hline hsa_circRNA_103901 & $<0.001$ & -36.919 & -5.206 & Down & Exonic & $\operatorname{chr} 5$ & - & uc003kfo.3 & LHFPL2 \\
\hline hsa_circRNA_100061 & 0.004 & -19.228 & -4.265 & Down & Exonic & chr1 & - & uc001aub.3 & DHRS3 \\
\hline hsa_circRNA_104046 & 0.003 & -20.371 & -4.348 & Down & Exonic & chr6 & + & uc003mtz.3 & WRNIP1 \\
\hline hsa_circRNA_104351 & 0.040 & -9.361 & -3.226 & Down & Exonic & chr7 & - & uc011kbg.2 & GLI3 \\
\hline hsa_circRNA_104601 & 0.035 & -12.922 & -3.691 & Down & Exonic & chr8 & - & uc003xpe. 3 & SLC20A2 \\
\hline hsa_circRNA_102489 & $<0.001$ & -35.219 & -5.138 & Down & Exonic & $\operatorname{chr} 19$ & + & uc002nkf.3 & UPF1 \\
\hline hsa_circRNA_102471 & 0.041 & -8.065 & -3.011 & Down & Exonic & $\operatorname{chr} 19$ & + & uc002nfj.1 & MYO9B \\
\hline hsa_circRNA_104280 & 0.002 & -21.819 & -4.447 & Down & Exonic & $\operatorname{chr} 7$ & - & uc003six.1 & PRKAR1B \\
\hline hsa_circRNA_101657 & 0.038 & -9.248 & -3.209 & Down & Exonic & $\operatorname{chr} 15$ & + & uc010urq.2 & IGF1R \\
\hline hsa_circRNA_104318 & 0.032 & -11.642 & -3.541 & Down & Exonic & $\operatorname{chr} 7$ & - & uc003sti.3 & ANKMY2 \\
\hline hsa_circRNA_102212 & 0.038 & -9.358 & -3.226 & Down & Exonic & $\operatorname{chr} 17$ & - & uc002jwc.1 & USP36 \\
\hline hsa_circRNA_100752 & 0.007 & -24.319 & -4.604 & Down & Exonic & $\operatorname{chr} 11$ & - & uc001maq.2 & OR51B5 \\
\hline hsa_circRNA_104190 & 0.002 & -23.098 & -4.529 & Down & Exonic & chr6 & - & uc003qez.2 & HBS1L \\
\hline hsa_circRNA_001369 & 0.024 & -10.828 & -3.436 & Down & Antisense & $\operatorname{chr} 12$ & - & NM_000020 & ACVRL1 \\
\hline hsa_circRNA_100677 & 0.005 & -21.387 & -4.418 & Down & Exonic & chr10 & - & uc009xxl.3 & PCGF6 \\
\hline
\end{tabular}

FC, fold change; circRNA, circular RNA; chrom, chromosome.

Reverse transcription-PCR validation of some differentially expressed circRNAs. We set a threshold as $\log _{2}$ fold-change $>5$ in upregulated circRNAs, $>3$ in downregulated circRNAs as the previous study (17), and P-value $<0.05$, and found 29 upregulated (Table II) and 28 downregulated differentially expressed circRNAs (Table III). Next, we randomly selected 20 differentially expressed circRNAs, including 10 upregulated $(102713,100715,100261,100924,104804,104707$, $102830,102298,100911$, and 102293) and 10 downregulated circRNAs (103538, 104318, 103477, 104280, 100144, 104984, $001369,103901,100677$, and 102464) for substantiation in the gastric tissue samples. The results of the microarray were in agreement with those of the real-time PCR; 6 selected upregulated circRNAs (Fig. 4A) and 5 selected downregulated circRNAs (Fig. 4B) were verified. However, the expressions of upregulated (Fig. 4C) and downregulated circRNAs (Fig. 4D) were not related to the expression of the host genes.

\section{Discussion}

Human gastric cancer is one of the most commonly known malignancies all over the world. A large number of studies have shown that the occurrence of gastric cancer involves several molecular mechanisms. However, the precise biological process of gastric cancer is not yet clearly elucidated. Several circRNAs have been recently discovered constituting a new specific class of endogenous non-coding RNAs. Hsa_circ_001569 promoted colorectal cancer in cell proliferation and invasion as a sponge of miR-145 (18). On the contrary, hsa_circ_002059 was found to be significantly down-regulated in gastric cancer as a typical circRNA, and its expression level was correlated with tumor metastasis and TNM stage (19). Thus, circRNAs might play a major role in the occurrence and development of gastric cancer; however, our understanding about the correlation between circRNAs expression and gastric cancer remains controversial due to the limited number of studies. Hence, the expression 
A

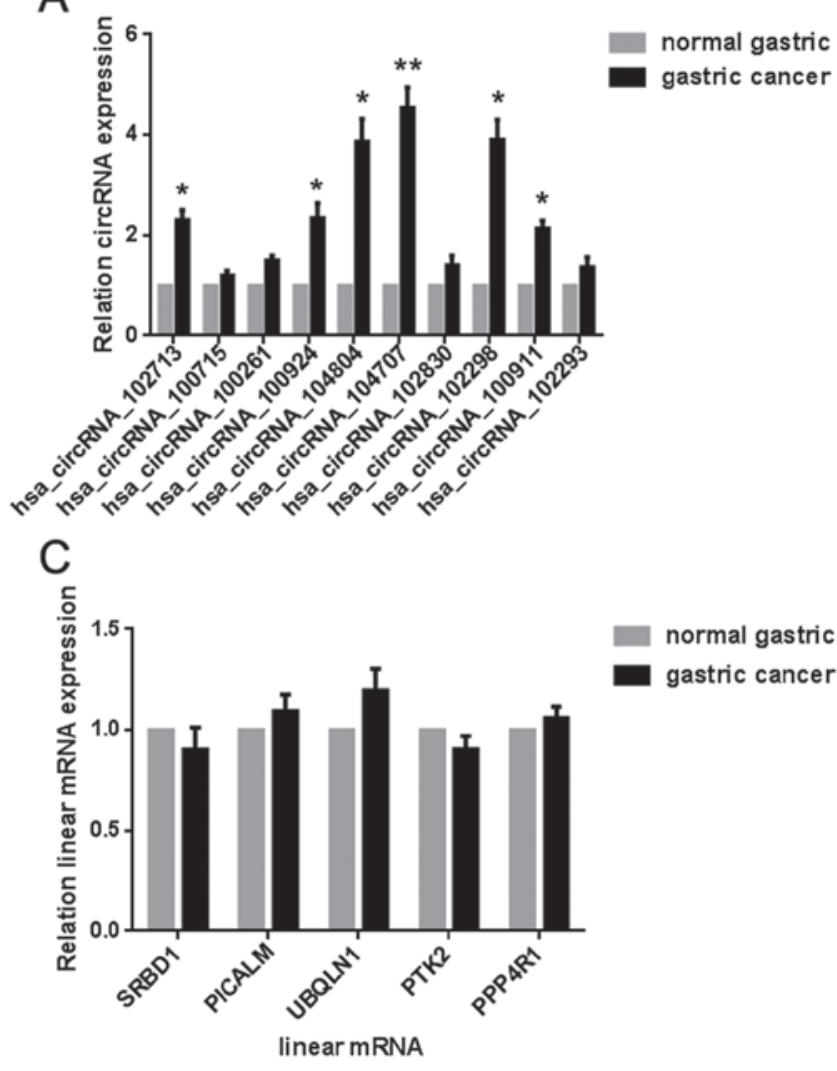

B

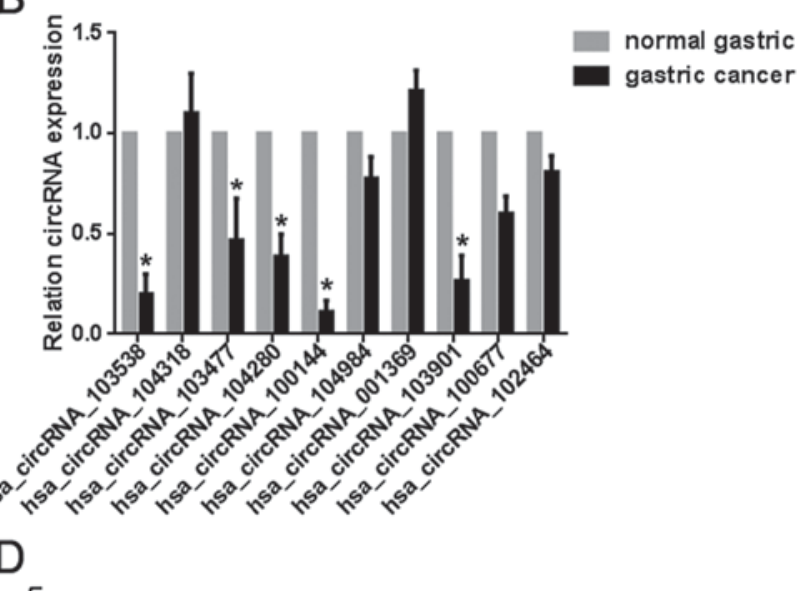

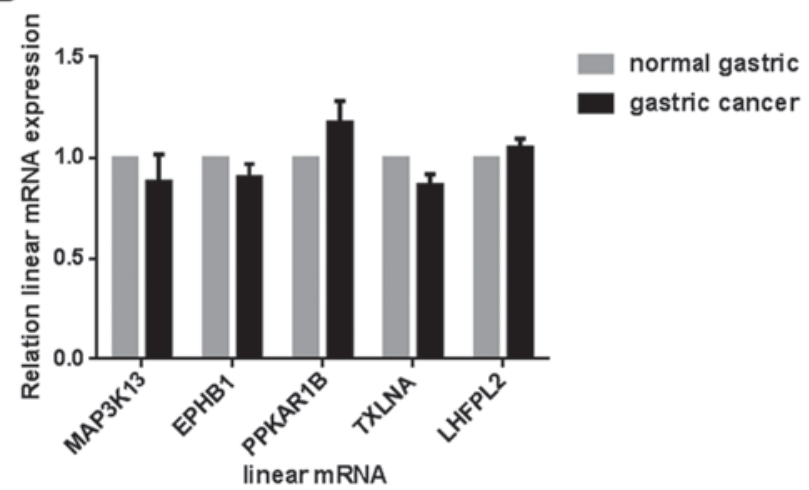

Figure 4. RT-PCR validation of some differentially expressed circRNAs and mRNAs from microarray analysis. (A) Selected upregulated circRNAs were verified by RT-PCR. (B) Selected downregulated circRNAs were verified by RT-PCR. (C) The relationship between the expression of upregulated circRNAs and host genes. (D) The relationship between the expression of downregulated circRNAs and host genes. Data shown are representative of 3 gastric cancer and 3 adjacent normal gastric tissues. Values are the mean \pm standard deviation. ${ }^{*} \mathrm{P}<0.05,{ }^{* * *} \mathrm{P}<0.01$ vs. normal gastric tissue group. circRNA, circular RNA; RT-PCR, reverse transcription polymerase chain reaction.

profile of circRNAs in gastric cancer necessitates further exploration with respect to the potential mechanisms.

The specific repeated pattern of chromosomal aberrations is not associated with tumorigenesis and progression in gastric cancer. The current study conformed to the conclusion considering that all the chromosomes can experience unequable changes (20). However, there are inconsistencies regarding the chromosomal location of dysregulated circRNAs. Our experimental results revealed that chromosomal abnormalities were mainly distributed on chromosomes $1,2,3,6,9,11$, and 17, while previous studies designated chromosomes $8,12,15,17$, and 20 in gastric cancer $(21,22)$. This phenomenon might be attributed to the following: firstly, the experimental method to detect chromosomal dysregulation in the past was FISH technology, while currently gene microarray is employed. Secondly, the sample size in the current study was small due to the high cost of gene microarray technology. Finally, the inherent differences in gastric cancer, such as the degree of pathological differentiation, stages, and grades, might also result in the differential distribution of chromosomal abnormalities.

Here, we reverse transcription-PCR verified the microarray analysis results. Recent evidence demonstrated that circRNAs play a crucial role in fine-tuning the level of miRNA-mediated regulation of gene expression via miRNA sequestration $(23,24)$. In addition, several of the predicted binding sites of circRNAs on miRNAs are functional and appear to be under less selective pressure as compared to the corresponding miRNA binding sites in mRNAs (25). However, in the current study, the expression of circRNAs did not correlate with the expression of the host genes, suggesting an independent regulation of transcription vs. circRNA formation. We will verify the findings in further studies with larger sample size. Combining with previous studies $(26,27)$, we currently propose that the circRNA-miRNA-mRNA axis may be the putative mechanism promoting the growth of the tumor, although the specific effect might not be deduced. Thus, further studies are essential for an insight into the exact mechanism.

In conclusion, we reported the profile of differentially expressed circRNAs between normal gastric and gastric cancer tissues. The network of differentially expressed numerous circRNAs was constructed and they found to be involved in the development and metabolism of gastric cancer with our and previous studies (27-29). Therefore, a further exploration of the biological processes and molecular mechanisms of the dysregulated circRNAs is imperative in order to clarify the pathogenesis of gastric cancer or provide a new therapeutic target via the regulation of the key circRNAs.

\section{Acknowledgements}

The authors would like to thank Dr Tianwei Gu for his linguistic assistance. 


\section{Funding}

The present study was supported by the Outstanding Youth Project of Nanjing City (grant no. JQX14005) and the Medjaden Academy \& Research Foundation for Young Scientists (Grant no. MJR20170029).

\section{Availability of data and materials}

All data generated or analyzed during the study are included in this published article.

\section{Authors' contributions}

$\mathrm{XZ}$ and XL designed the research. YS, JZ, ZF, BZ, MC, XL and $\mathrm{XZ}$ performed the experiments. YS, JZ, BZ and MC analyzed the data. YS wrote the paper and ZF, XL and XZ critically revised the manuscript for important intellectual content.

\section{Ethics approval and consent to participate}

The present study was approved by the Research Ethics Committee of the Affiliated Drum Tower Hospital of Nanjing University, Medical School (Nanjing, China) and all patients provided informed consent prior to their inclusion within the study.

\section{Consent for publication}

All patients provided written informed consent for the publication of their data.

\section{Competing interests}

The authors declare that they have no competing interests.

\section{References}

1. Bou Kheir T, Futoma-Kazmierczak E, Jacobsen A, Krogh A, Bardram L, Hother C, Grønbæk K, Federspiel B, Lund AH and Friis-Hansen L: miR-449 inhibits cell proliferation and is down-regulated in gastric cancer. Mol Cancer 10: 29, 2011.

2. Tan P and Yeoh KG: Genetics and molecular pathogenesis of gastric adenocarcinoma. Gastroenterology 149: 1153-1162.e3, 2015

3. Lochhead P and El-Omar EM: Gastric cancer. Br Med Bull 85: 87-100, 2008.

4. Nigro JM, Cho KR, Fearon ER, Kern SE, Ruppert JM, Oliner JD, Kinzler KW and Vogelstein B: Scrambled exons. Cell 64: 607-613, 1991

5. Hansen TB, Jensen TI, Clausen BH, Bramsen JB, Finsen B, Damgaard CK and Kjems J: Natural RNA circles function as efficient microRNA sponges. Nature 495: 384-388, 2013.

6. Memczak S, Jens M, Elefsinioti A, Torti F, Krueger J, Rybak A, Maier L, Mackowiak SD, Gregersen LH, Munschauer M, et al: Circular RNAs are a large class of animal RNAs with regulatory potency. Nature 495: 333-338, 2013.

7. Jeck WR, Sorrentino JA, Wang K, Slevin MK, Burd CE, Liu J, Marzluff WF and Sharpless NE: Circular RNAs are abundant, conserved, and associated with ALU repeats. RNA 19: 141-157, 2013.

8. Guo JU, Agarwal V, Guo H and Bartel DP: Expanded identification and characterization of mammalian circular RNAs. Genome Biol 15: 409, 2014

9. Wang PL, Bao Y, Yee MC, Barrett SP, Hogan GJ, Olsen MN, Dinneny JR, Brown PO and Salzman J: Circular RNA is expressed across the eukaryotic tree of life. PLoS One 9: e90859, 2014.
10. Reddy SD, Ohshiro K, Rayala SK and Kumar R: MicroRNA-7, a homeobox D10 target, inhibits p21-activated kinase 1 and regulates its functions. Cancer Res 68: 8195-8200, 2008.

11. Webster RJ, Giles KM, Price KJ, Zhang PM, Mattick JS and Leedman PJ: Regulation of epidermal growth factor receptor signaling in human cancer cells by microRNA-7. J Biol Chem 284: 5731-5741, 2009.

12. Saydam O, Senol O, Würdinger T, Mizrak A, Ozdener GB, Stemmer-Rachamimov AO, Yi M, Stephens RM, Krichevsky AM, Saydam N, et al: miRNA-7 attenuation in schwannoma tumors stimulates growth by upregulating three oncogenic signaling pathways. Cancer Res 71: 852-861, 2011.

13. Fang Y, Xue JL, Shen Q, Chen J and Tian L: MicroRNA-7 inhibits tumor growth and metastasis by targeting the PI3K/AKT pathway in hepatocellular carcinoma. Hepatology 55: 1852-1862, 2012.

14. Xiong S, Zheng Y, Jiang P, Liu R, Liu X and Chu Y: MicroRNA-7 inhibits the growth of human non-small cell lung cancer A549 cells through targeting BCL-2. Int J Biol Sci 7: 805-814, 2011.

15. Zhang N, Li X, Wu CW, Dong Y, Cai M, Mok MT, Wang H, Chen J, Ng SS, Chen M, et al: microRNA-7 is a novel inhibitor of YY1 contributing to colorectal tumorigenesis. Oncogene 32: 5078-5088, 2013.

16. Li F, Zhang L, Li W, Deng J, Zheng J, An M, Lu J and Zhou Y: Circular RNA ITCH has inhibitory effect on ESCC by suppressing the Wnt/ $\beta$-catenin pathway. Oncotarget 6: 6001-6013, 2015.

17. Wu HJ, Zhang CY, Zhang S, Chang M and Wang HY: Microarray expression profile of circular RNAs in heart tissue of mice with myocardial infarction-induced heart failure. Cell Physiol Biochem 39: 205-216, 2016.

18. Xie H, Ren X, Xin S, Lan X, Lu G, Lin Y, Yang S, Zeng Z, Liao W, Ding YQ and Liang L: Emerging roles of circRNA_001569 targeting miR-145 in the proliferation and invasion of colorectal cancer. Oncotarget 7: 26680-26691, 2016.

19. Li P, Chen S, Chen H, Mo X, Li T, Shao Y, Xiao B and Guo J: Using circular RNA as a novel type of biomarker in the screening of gastric cancer. Clin Chim Acta 444: 132-136, 2015.

20. Luo H, Zhao X, Wan X, Huang S and Wu D: Gene microarray analysis of the IncRNA expression profile in human urothelial carcinoma of the bladder. Int J Clin Exp Med 7: 1244-1254, 2014

21. Cheng L, Zhang Q, Yang S, Yang Y, Zhang W, Gao H, Deng X and Zhang Q: A 4-gene panel as a marker at chromosome 8q in Asian gastric cancer patients. Genomics 102: 323-330, 2013.

22. Noguchi T, Wirtz HC, Michaelis S, Gabbert HE and Mueller W: Chromosomal imbalances in gastric cancer. Correlation with histologic subtypes and tumor progression. Am J Clin Pathol 115: 828-834, 2001

23. Andrés-León E, Núñez-Torres R and Rojas AM: miARma-Seq: A comprehensive tool for miRNA, mRNA and circRNA analysis. Sci Rep 6: 25749, 2016.

24. Caiment F, Gaj S, Claessen S and Kleinjans J: High-throughput data integration of RNA-miRNA-circRNA reveals novel insights into mechanisms of benzo[a]pyrene-induced carcinogenicity. Nucleic Acids Res 43: 2525-2534, 2015.

25. Mu XJ, Lu ZJ, Kong Y, Lam HY and Gerstein MB: Analysis of genomic variation in non-coding elements using population-scale sequencing data from the 1000 Genomes Project. Nucleic Acids Res 39: 7058-7076, 2011.

26. Li W,Zhong C, Jiao J, Li P, Cui B, Ji C and Ma D: Characterization of hsa_circ_0004277 as a new biomarker for acute myeloid leukemia via circular RNA profile and bioinformatics analysis. Int J Mol Sci 18: pii: E597, 2017.

27. Dang Y, Ouyang X, Zhang F, Wang K, Lin Y, Sun B, Wang Y, Wang $L$ and Huang Q: Circular RNAs expression profiles in human gastric cancer. Sci Rep 7: 9060, 2017.

28. Shao Y, Li J, Lu R, Li T, Yang Y, Xiao B and Guo J: Global circular RNA expression profile of human gastric cancer and its clinical significance. Cancer Med 6: 1173-1180, 2017.

29. Sui W, Shi Z, Xue W, Ou M, Zhu Y, Chen J, Lin H, Liu F and Dai Y: Circular RNA and gene expression profiles in gastric cancer based on microarray chip technology. Oncol Rep 37: 1804-1814, 2017. 